\title{
The turnstile mechanism across the Kuroshio current: analysis of dynamics in altimeter velocity fields
}

\author{
C. Mendoza ${ }^{1}$, A. M. Mancho ${ }^{1}$, and M.-H. Rio ${ }^{2}$ \\ ${ }^{1}$ Instituto de Ciencias Matemáticas, CSIC-UAM-UC3M-UCM, Serrano 121, 28006 Madrid, Spain \\ ${ }^{2} \mathrm{CLS}$ - Space Oceanography Division, Toulouse, France
}

Received: 30 September 2009 - Revised: 2 February 2010 - Accepted: 10 February 2010 - Published: 11 March 2010

\begin{abstract}
In this article we explore the utility of dynamical systems tools for visualizing transport in oceanic flows described by data sets measured from satellites. In particular we have found the geometrical skeleton of some transport processes in the Kuroshio region. To this end we have computed the special hyperbolic trajectories, and identified them as distinguished hyperbolic trajectories, that act as organizing centres of the flow. We have computed their stable and unstable manifolds, and they reveal that the turnstile mechanism is at work during several spring months in the year 2003 across the Kuroshio current. We have found that near the hyperbolic trajectories takes place a filamentous transport frontcross the current that mixes waters from both sides.
\end{abstract}

\section{Introduction}

Oceanic transport processes have an important impact on the global climate (Bower et al., 2009; Katija \& Dabiri, 2009), as oceans are important reservoirs of heat and carbon dioxide which are the most important driving forces of climate change. Understanding and describing oceanic transport processes has been a challenging task. Great ocean currents such as the Gulf Stream or the Kuroshio current that in their Eulerian description seem smooth, river-like streams, present a messy pattern when examined using float tracking (Bower et al., 2009; Lavender et al., 2000). Understanding such patterns is essential for predicting the effects of global warming on the Earth's future climate.

In recent years, dynamical systems theory has provided a framework for describing transport in time dependent flows. It has been useful for finding transport routes in complex flows, which means it has successfully found ordering structures from erratic patterns. Typical tools in dynamical systems theory are Lyapunov exponents and invariant manifolds. These notions are defined only for infinite time sys-

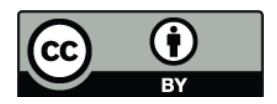

Correspondence to: A. M. Mancho (a.m.mancho@icmat.es) tems. However, realistic flows like those arising in geophysics or oceanography, are not infinite time systems, and for their description, finite time versions of the definition of Lyapunov exponents, such as finite size Lyapunov exponents FSLE (Aurell et al., 1997) and finite time Lyapunov exponents FTLE (Nese, 1989; Haller, 2001) are used. Invariant manifolds are objects that in the classical theory of dynamical systems are asymptotically defined for special trajectories such as fixed points or periodic orbits with hyperbolic stability. Recent articles by Ide et al. (2002); Ju et al. (2003); Madrid and Mancho (2009) have provided definitions that generalise these reference orbits for time dependent aperiodic flows. These special trajectories are called distinguished trajectories. The definition given in (Madrid and Mancho, 2009 ) is proven to include fixed points and periodic orbits as particular cases. Separation points (SP) on boundaries, such as detachment and reattachment points are also examples of distinguished hyperbolic trajectories (DHT). The computation of SP has been also addressed in (Haller, 2004). However, the definition provided in (Madrid and Mancho, 2009) provides a systematic way for finding these special trajectories not only on boundaries but also in the interior of the flow. DHT and their stable and unstable manifolds are the essential dynamical tools we will apply in this article for describing transport in geophysical flows.

Lagrangian and Eulerian observational datasets have held great promise for providing insight on oceanic transport. Drifter observations have revealed fluid exchange (Bower and Rossby, 1989; Lozier et al., 2003) in the Gulf Stream. An important step forward in the description of the exchange of trajectories across the Gulf Stream was the meandering kinematic model proposed by Bower (1991). Studies such as (Lozier et al., 2003) have succeeded in describing transport in simplified models of the Gulf Stream by means of lobe dynamics (Bower, 1991). Recently, ocean-observing satellites are entering a new operational era and naturally occupy an important place in programs designed to manage and predict ocean and climate change. These instruments may provide almost real time processing of satellite

Published by Copernicus Publications on behalf of the European Geosciences Union and the American Geophysical Union. 
altimeter data. In this article we describe cross-frontal transport along a major ocean current, the Kuroshio current, based on a Lagrangian description of observed altimeter velocities, over a period of 54 days in 2003. Our description uses dynamical objects such as the stable and unstable manifolds of distinguished trajectories. We detect the turnstile mechanism across a part of the Kuroshio current. The turnstile survives persistently from 3 April to 26 May 2003. This mechanism had already been found to be present in realistic numerically produced data sets modeling the North Balearic front (Mancho et al., 2008). In this article we discuss similar ideas in the context of dealing with altimeter data. Finite time Lyapunov exponents methods used to compute transport processes in geophysical flows is referred to as Lagrangian Coherent Structures (LCS). Recently LCS and FSLE have been applied to describe dynamics in altimeter datasets (BeronVera et al., 2008; Rossi et al., 2008; Rypina et al., 2009), although these studies are focused on areas of the ocean surface different from the Kuroshio current.

The structure of the article is as follows. In Sect. 2 we describe the data analysed and to what extent the velocity field satisfies the divergence free approach. Section 3 describes the details of the interpolation used on the data and provides the equations of the continuous dynamical system used in our analysis. Section 4 describes the computation of the distinguished hyperbolic trajectories and of their stable and unstable manifolds for the system built in Sect. 3. Section 5 describes the turnstile mechanism governing the dynamics across a current that persist on the data amid latitudes $32^{\circ} \mathrm{N}$ and $40^{\circ} \mathrm{N}$ and longitudes $155^{\circ} \mathrm{E}$ and $165^{\circ} \mathrm{E}$ during the months of April to May 2003. Finally Sect. 6 details the conclusions.

\section{The altimeter dataset}

In this work we analyze transport across the Kuroshio current from 3 April to 26 May 2003, spanning a time interval of 54 days.

We use daily maps of surface currents computed at CLS in the framework of the SURCOUF project (Larnicol et al., 2006) from a combination of altimetric sea surface heights and windstress data in a two-steps procedure: on the one hand, multimission (ERS-ENVISAT, TOPEX-JASON) altimetric maps of sea level anomaly (SLA) are added to the RIO05 global Mean Dynamic Topography (Rio and Hernandez, 2004; Rio et al., 2005) to obtain global maps of sea surface heights from which surface geostrophic velocities $\left(u_{\mathrm{g}}\right.$, $v_{\mathrm{g}}$ ) are obtained by simple derivation.

$$
\begin{gathered}
u_{\mathrm{g}}=-\frac{g}{f} \frac{\partial h}{\partial y} \\
v_{\mathrm{g}}=\frac{g}{f} \frac{\partial h}{\partial x}
\end{gathered}
$$

where $f$ is the Coriolis parameter and $g$ the gravitational constant.
On the other hand, the Ekman components of the ocean surface current $\left(u_{\mathrm{ek}}, v_{\mathrm{ek}}\right)$ is estimated using a 2-parameter model: $u_{\mathrm{ek}}=b e^{i \theta} \tau$ where $b$ and $\theta$ are estimated by latitudinal bands from a least square fit between ECMWF 6-hourly windstress analysis $\tau$ and an estimate of the Ekman current obtained removing the altimetric geostrophic current from the total current measured by drifting buoy velocities available from 1993 to 2005 (the method is described in further details in (Rio and Hernandez, 2003)).

Both the geostrophic and the Ekman component of the ocean surface current are added to obtain estimates of the ocean total surface current that are used in the Kuroshio area for the present study.

Figure 1 shows a sample of the velocity field on 4 April 2003. We have marked with a line the current across which we study transport. The presence in the nearby of two counterclockwise eddies suggests the presence of two hyperbolic points at both sides of the line that could maintain a turnstile mechanism. We will show in Sect. 5 that this is truly the case. The interactions of these type of eddies with the main current has been shown to trigger the Kuroshio meander formation (Waseda et al., 2003).

Our work considers that in the area under study the particle motion is restricted to the ocean surface, i.e, there is no significant vertical motion. The altimeter-derived velocity field is two-dimensional. However, since the Ekman correction has been introduced, it is not guaranteed that the data is divergence free. We have computed the divergence of the velocity field in the whole period and area under study and we have verified that despite these corrections the divergence free approach is still valid.

\section{The equations of motion}

The equations of motion that describe the horizontal evolution of particle trajectories on a sphere are

$$
\begin{aligned}
& \frac{d \phi}{d t}=\frac{u(\phi, \lambda, t)}{R \cos (\lambda)}, \\
& \frac{d \lambda}{d t}=\frac{v(\phi, \lambda, t)}{R},
\end{aligned}
$$

where $u$ and $v$ represent respectively the eastward and northward components of the altimetry surface velocity field described in the previous section. The particle trajectories must be integrated in Eqs. (3)-(4) and since information is provided just in a discrete space-time grid, the first issue to deal with is that of interpolation of discrete data sets. A recent paper by Mancho et al. (2006) compares different interpolation techniques in tracking particle trajectories. Bicubic spatial interpolation in space (Press et al., 1992) and third order Lagrange polynomials in time are shown to provide a computationally efficient and accurate method. We use this technique in our computations. However we notice that bicubic spatial interpolation in space as discussed in Press et al. (1992) requires a uniformly spaced grid. Our data input is expressed 


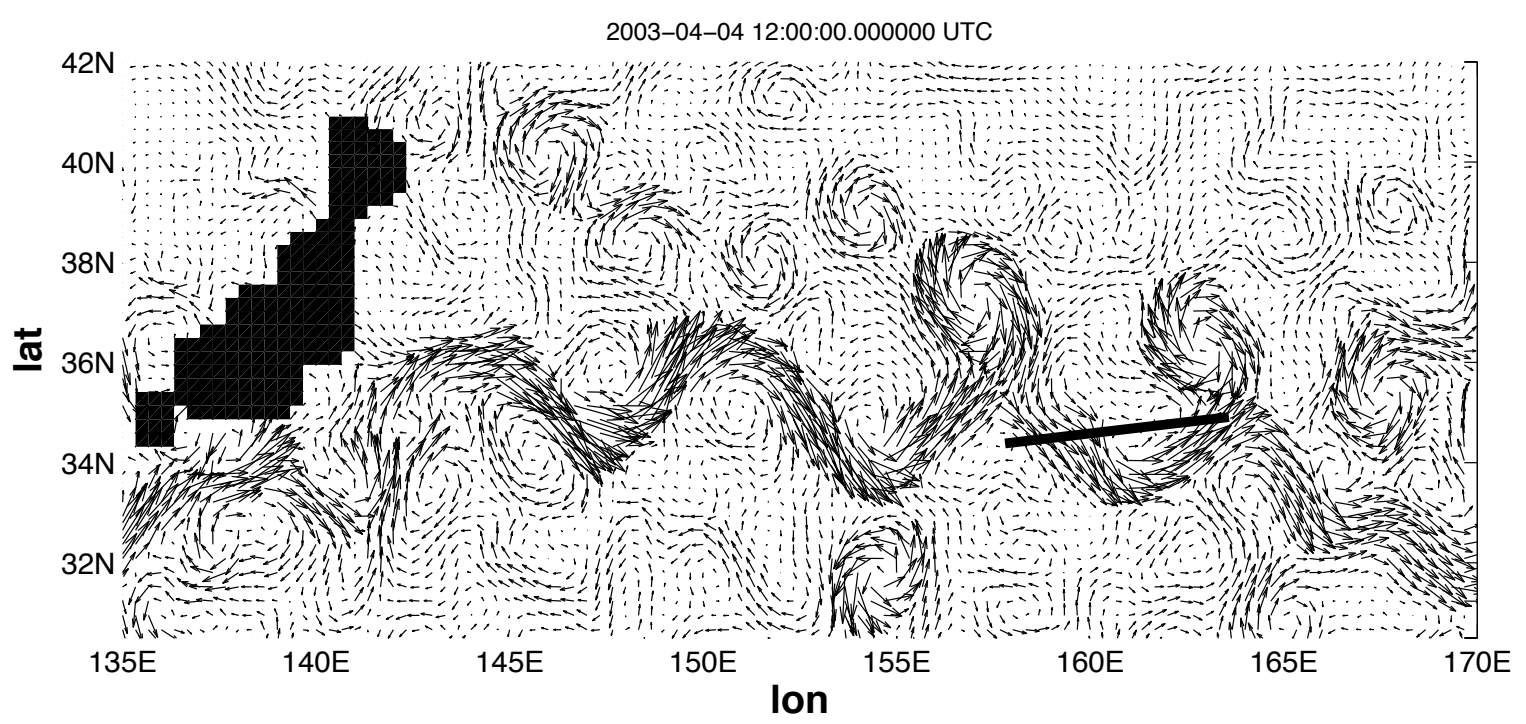

Fig. 1. Velocity field of the Kuroshio current on 4 April 2003. A line marks a piece of current across which transport is studied. Maximum values of the velocity field are about $3.65 \mathrm{~m} / \mathrm{s}$.

in spherical coordinates, and the grid is not uniformly spaced in the latitude coordinate. In order to interpolate in a uniformly spaced grid, we transform our coordinate system $(\lambda$, $\phi)$ to a new one $(\mu, \phi)$. The latitude $\lambda$ is related to the new coordinate $\mu$ by

$\mu=\ln |\sec \lambda+\tan \lambda|$

Our velocity field is now on a uniform grid in the $(\mu, \phi)$ coordinates. The equations of motion in the new variables are

$$
\begin{aligned}
\frac{d \phi}{d t} & =\frac{u(\phi, \mu, t)}{R \cos (\lambda(\mu))} \\
\frac{d \mu}{d t} & =\frac{v(\phi, \mu, t)}{R \cos (\lambda(\mu))}
\end{aligned}
$$

where $\lambda(\mu)$ is obtained by inverting Eq. (5), i.e.

$\lambda=\frac{\pi}{2}-2 \arctan \left(e^{-\mu}\right)$.

Once trajectories are integrated using Eqs (6)-(7), for presentation purposes one can convert $\mu$ values back to latitudes $\lambda$ by using (8).

\section{Distinguished hyperbolic trajectories and manifolds}

Distinguished hyperbolic trajectories and their unstable and stable manifolds are the dynamical systems objects used to describe and quantify transport.

Figure 1 marks with a line the eulerian feature across which we study transport. The current, similarly to that studied in (Mancho et al., 2008; Coulliette and Wiggins, 2001), flows eastward. We identify a DHT in the western part of the flow, for which we compute the unstable manifold, and a DHT in the eastern part, for which we compute the stable manifold.

Computation of distinguished hyperbolic trajectories for aperiodic flows has been discussed in Ide et al. (2002); Ju et al. (2003); Mancho et al. (2004); Madrid and Mancho (2009). The approach taken in this article is that of (Madrid and Mancho, 2009), which is based on the function $M$ defined as follows. Let $(\phi(t), \mu(t))$ denote a trajectory of the system (6)(7). For all initial conditions $\left(\phi^{*}, \mu^{*}\right)$ in an open domain $\mathcal{B}$ of the ocean surface, at a given time $t^{*}$, consider the function $M\left(\phi^{*}, \mu^{*}, t^{*}\right)_{\tau}: \mathcal{B} \cdot t \rightarrow \mathbb{R}$ defined by,

$$
M=\int_{t^{*}-\tau}^{t^{*}+\tau} \sqrt{\left(\frac{d \phi(t)}{d t}\right)^{2}+\left(\frac{d \mu(t)}{d t}\right)^{2}} d t,
$$

For an initial condition $\left(\phi^{*}, \mu^{*}\right)$ at $t^{*}$, the function $M$ measures the length of the curve outlined on the plane $(\phi, \mu)$ by a trajectory from $t^{*}-\tau$ to $t^{*}+\tau$.

We discuss in more detail the numerical evaluation of $M$ as defined in Eq. (9). Trajectories $(\phi(t), \mu(t))$ of the system (6)-(7) are obtained numerically, and thus represented by a finite number of points, $L$. A discrete version of Eq. (9) is:

$$
M=\sum_{j=1}^{L-1}\left(\int_{p_{i}}^{p_{f}} \sqrt{\left(\frac{d \phi_{j}(p)}{d p}\right)^{2}+\left(\frac{d \mu_{j}(p)}{d p}\right)^{2}} d p\right),
$$

where the functions $\phi_{j}(p)$ and $\mu_{j}(p)$ represent a curve interpolation parametrized by $p$, and the integral

$\int_{p_{i}}^{p_{f}} \sqrt{\left(\frac{d \phi_{j}(p)}{d p}\right)^{2}+\left(\frac{d \mu_{j}(p)}{d p}\right)^{2}} d p$

is computed numerically. Following the methodology described in (Madrid and Mancho, 2009) we have used the interpolation method used by Dritschel (1989) in the context 
(a)

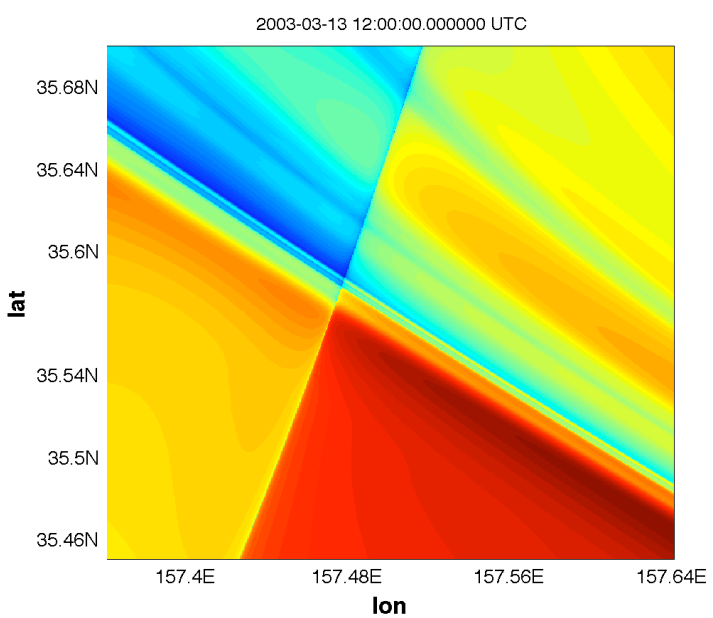

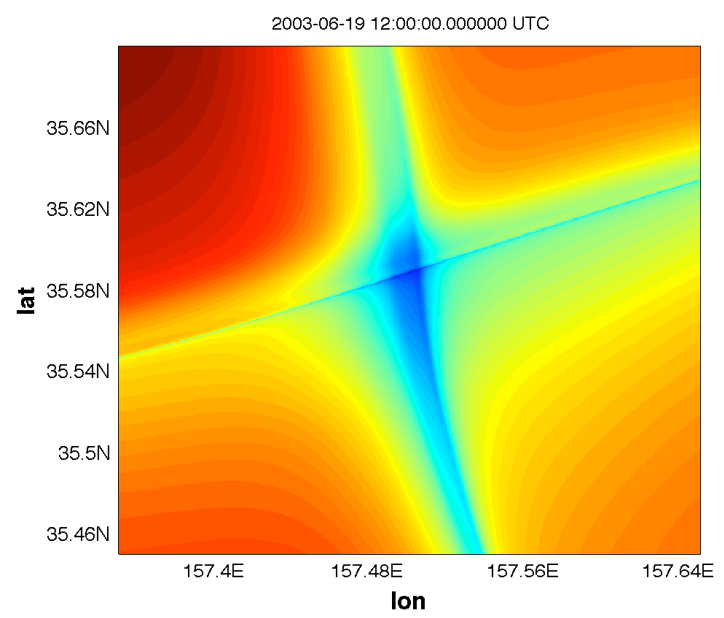

Fig. 2. Structure of the function $M$ for $\tau=15$, (a) in the neighbourhood of the western DHT, DHTW, on 13 March 2003 ; and (b) in the neighbourhood of the eastern DHT, DHT $\mathrm{E}$, on 19 June 2003.

of contour dynamics. To compute the integral (11) we have used the Romberges method (see Press et al., 1992) of order $2 K$ with $K=5$.

The function $M$ is defined over an open set, so it does not necessarily attain a minimum, but if it does, the minimum is denoted by $\min \left(M_{\tau}\right)$. In (Madrid and Mancho, 2009) there is found the position of the minimum of $M$ for each fixed $t^{*}$ as a function of $\tau$. For $\tau \gg 0$, the minimum converges to a fixed value called the limit coordinate. Distinguished trajectories are those that for a time interval, pass close enough (at a distance $\epsilon$, typically within numerical accuracy) to a path of limit coordinates. Computing limit coordinates becomes a method for finding distinguished trajectories because it is quite typical that there exist trajectories passing near these paths.

We have computed limit coordinates on opposite sides of the line depicted in Fig. 1. A typical profile of the function $M$ is shown for $\tau=15$ in Fig. 2. The color code reads as follows: maximum $M$ values are dark red, while minima are dark blue. As discussed in (Mendoza and Mancho, 2010) the important information encoded by $M$ is discernible from its minima and its singular features, so no specific colorbar is required. Figure 2 a shows the contour plot near the western DHT, $\mathrm{DHT}_{\mathrm{W}}$, on 13 March 2003; Fig. 2b) depicts the contour plot of $M$ for the eastern DHT, DHT , on 19 June 2003. The time evolutions of two paths of limit coordinates, DHT $_{\mathrm{W}}$ and $\mathrm{DHT}_{\mathrm{W}}^{+}$, at the West are displayed in Fig. 3. Initial conditions for each limit path evolve staying near it for a certain time interval. However to prove that a trajectory stays close to the path over long time intervals is a difficult task because it is not possible to obtain the whole DHT by direct integration methods. As discussed in (Madrid and Mancho, 2009), DHTs are elusive and any initial condition in the neighbourhood of one, eventually leaves it through the unstable manifold. If the paths in Fig. 3 are trajectories they should satisfy Eqs. (6)-(7). However verifying this requires the computa- tion of the time derivative of the paths of limit coordinates and these computations are very inaccurate. A better choice for confirming their trajectory character is to verify that they are within a distance $\epsilon$ of the intersection of the stable and unstable manifolds. The trajectory $\mathrm{DHT}_{\mathrm{W}}$ remains distinguished from 5 to 11 May 2003. Outside this time interval, the path of limit coordinates although it still exists for a short period, is no longer a trajectory and eventually the path is lost. This kind of behavior coincides with that described in (Madrid and Mancho, 2009) for a similar DHT in a highly aperiodic flow. At the western end there exists a second DHT labelled as $\mathrm{DHT}_{\mathrm{W}}^{+}$, which remains distinguished in an approximately complementary time interval, between 10 May and 1 June 2003. The distinguished property of $\mathrm{DHT}_{\mathrm{W}}^{+}$ends similarly to that of $\mathrm{DHT}_{\mathrm{W}}$. The panels in Fig. 4 show the path of limit coordinates in the east. The trajectory $\mathrm{DHT}_{\mathrm{E}}$ remains distinguished between 25 March and 24 June 2003.

The fact that the paths of limit coordinates are close to trajectories, at least for a certain interval of time, indicate that the minima of $M$ provide Lagrangian information. When the motion described in Eqs. (6)-(7) is expressed in a new reference frame, trajectories are related to the ones in the old frame by the same coordinate transformation. A question then is: "Do trajectories in the new frame also have the distinguished property?". The answer is yes. An example is reported for instance in (Madrid and Mancho, 2009), where limit coordinates for the rotating and non-rotating Duffing equation are related by the appropriate coordinate transformation.

Once an appropriate DHT has been identified we compute its stable and unstable manifolds. A novel technique for computing stable and unstable manifolds of hyperbolic trajectories in aperiodic flows is developed by Mancho et al. (2003). In Mancho et al. $(2004,2008)$ this manifold computation is successfully applied to quite realistic flows. We will now apply these same algorithms to compute stable and unstable 
(a)

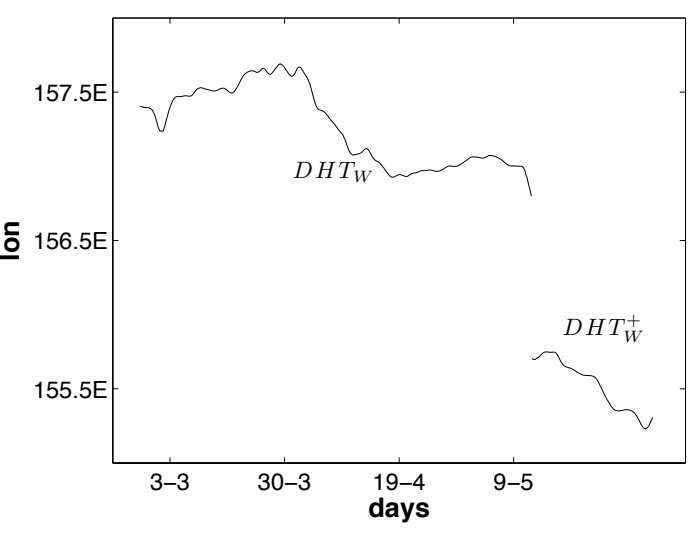

(b)

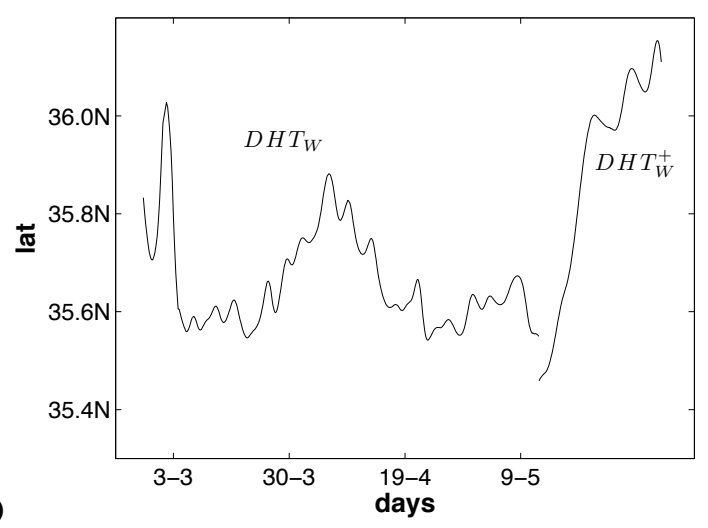

Fig. 3. Path of limit coordinates for $\mathrm{DHT}_{\mathrm{W}}$ and $\mathrm{DHT}_{\mathrm{W}}^{+}$; (a) longitude vs. time; and (b) latitude vs. time.

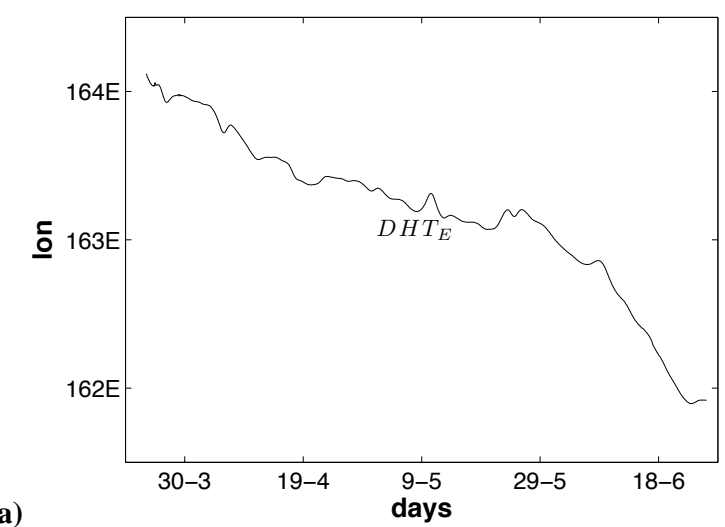

(a)

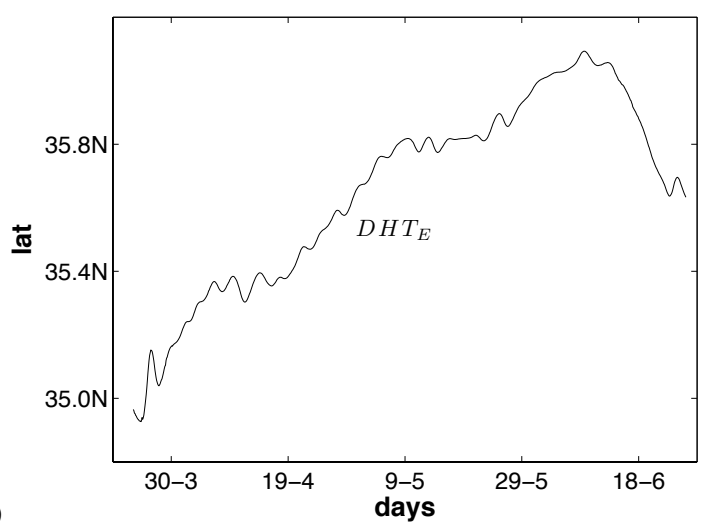

(b)

Fig. 4. Path of limit coordinates for $\mathrm{DHT}_{\mathrm{E}}$; (a) longitude vs. time; and (b) latitude vs. time.

manifolds of DHT found in altimeter data sets. In our study we compute the unstable manifold of $\mathrm{DHT}_{\mathrm{W}}$, and the stable manifold of $\mathrm{DHT}_{\mathrm{E}}$. The manifold computation requires as inputs the position of the DHT at a given time and also the direction of the unstable or stable subspace at that time. In this work we obtain this in a different manner than that proposed in Mancho et al. (2004). We take advantage of the structure of the function $M$ depicted in Fig. 2, which shows sharp features. In (Mendoza and Mancho, 2010) these lines are reported to be an advected structure of Eqs. (6)-(7), thus confirming they are aligned with the stable and unstable subspaces. We use these directions as input for the unstable or stable subspaces in the manifold algorithm.

Figure 5 shows the computed manifolds and DHTs on 4 May 2003. Almost every distinguishable line in this figure contains numerous foldings of each manifold, thus confirming how intricate they may be. In our computations the unstable manifold at a time $t_{*}$ is made of trajectories that at time $t_{0}, t_{0}<t_{*}$, were at a small segment aligned with the unstable subspace of the DHT. This is a finite time version of the asymptotic condition required for unstable manifolds in infinite time systems. Similarly the stable manifold at a time $t_{*}$ is made of trajectories that at time $t_{1}, t_{1}>t_{*}$ are in a small segment aligned with the stable subspace of the DHT. Other approaches such as FTLE or FSLE compute manifolds at a given time as ridges of a scalar field, providing pieces of curves that are material curves. However, links between pieces of curves are difficult to establish as they fade away. The intricate curves of Fig. 5 are linked curves (due to the asymptotic condition imposed in their computation) and this is an advantage over other methods. The manifolds in Fig. 5, however, are too complex to apply to concrete transport problems so that in next section we will extract from them pieces with the appropriate dynamical information.

\section{The turnstile mechanism}

The turnstile mechanism, extensively used and explained in the literature (Malhotra and Wiggins, 1998; Rom-Kedar et al., 1990), has been found to play a role in transport in several oceanographic contexts (Mancho et al., 2008; Coulliette and Wiggins, 2001). Our manifold computations reveal the presence of a turnstile mechanism transporting masses of water across the current of Fig. 1 from north to south and viceversa. We study it for a period of 54 days from 3 April to 26 May 2003. 


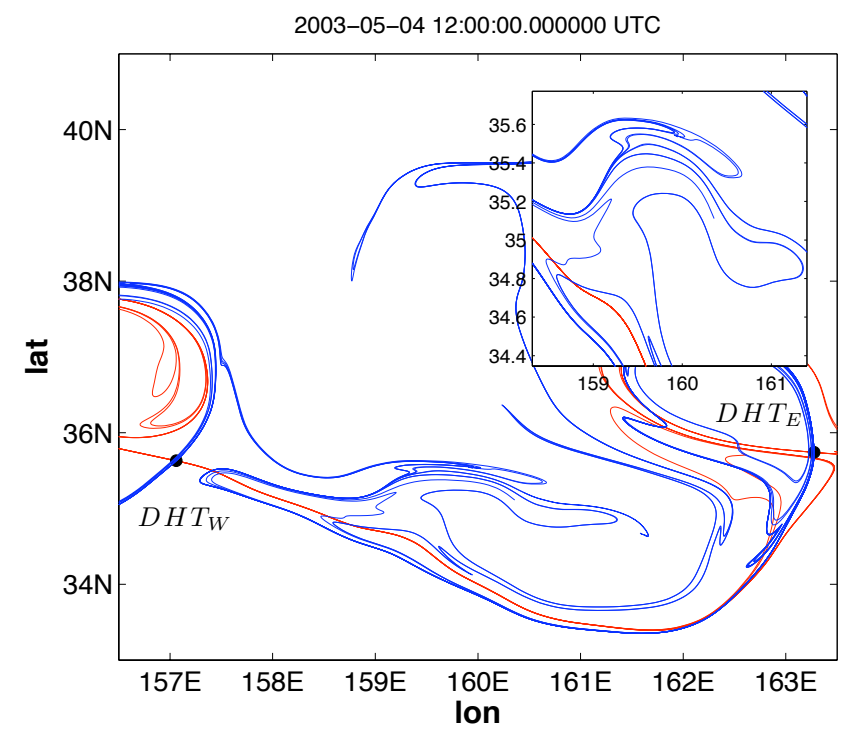

Fig. 5. Stable (blue) manifold of $\mathrm{DHT}_{\mathrm{E}}$ at the eastern end of the current and unstable (red) manifold of $\mathrm{DHT}_{\mathrm{W}}$ at the west.

First, we determine a time dependent Lagrangian barrier made of pieces of manifolds that separates north from south. Manifolds are made of trajectories. Thus, as no particle trajectories can cross them, they are barriers to transport. Figure 6 shows barriers at days 4 and 17 April, by depicting a piece of the unstable manifold of $\mathrm{DHT}_{\mathrm{W}}$ and a piece of the stable manifold of $\mathrm{DHT}_{\mathrm{E}}$. How should one choose these portions from intricate curves such as those depicted in Fig. 5? First, we consider that a manifold has two branches separated by the DHT, which is taken as a reference. The selections are curves of relatively short lengths either on one or both sides of the DHT. For instance, for the unstable manifold case, these curves are traced out by all the trajectories that expanded from a small segment along the unstable subspace of the DHT during a relatively short interval of time. If segments along the unstable subspace are expanded for longer time intervals, then the manifolds become as intricate as those in Fig. 5. In Fig. 6 the boundary intersection points are marked with letters $a$ and $b$. Intersection points satisfy the property of invariance, which means that if the stable and unstable manifolds intersect in a point at a given time, then they intersect for all time, and the intersection point is then a trajectory. In order to help us to understand the time evolution of lobes, we will depict the position of trajectories $a$ and $b$ at different times.

Figure 7 shows longer pieces of the unstable and stable manifolds at the same days selected in Fig. 6. Manifolds intersect forming regions called lobes. It is only the fluid that is inside the lobes that can participate in the turnstile mechanism. Figure 7 displays two snapshots showing the evolution of lobes from 2 to 17 April. There it can be seen how the lobe which is at the north of the barrier at 2 April crosses to the south of the barrier on 17 April. Similarly the lobe which is at the south on 2 April, crosses to the north on 17 April. Trajectories $a$ and $b$ are depicted showing that they evolve, approaching $\mathrm{DHT}_{\mathrm{E}}$. The area surrounded by a lobe has been coloured with green if near the western DHT, $\mathrm{DHT}_{\mathrm{W}}$, it was on the north side, and magenta if it was on the south. The turnstile mechanism transports magenta to the north and green to the south.

Along the time interval under study, several lobes are formed mixing waters of north and south. Figure 8 contains a time sequence showing the evolution of several lobes created by the intersection of the stable and unstable manifolds. A sequence of trajectories $a, b, c, \ldots$ on the intersection points is depicted. These trajectories evolve from west to east and serve as references for tracking lobe evolution. The lobes between the intersection trajectories $a$ and $b$ of Fig. 8a are not depicted in Fig. 8b as they are very thin filaments stretched along the $\mathrm{DHT}_{\mathrm{E}}$. In the same way the lobes between the intersection trajectories $c$ and $d$ of Fig. $8 \mathrm{~b}$ are not depicted in Fig. 8a as they are very thin filaments stretched

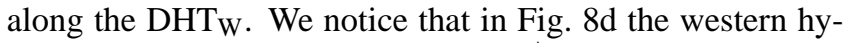
perbolic trajectory is labelled as $\mathrm{DHT}_{\mathrm{W}}^{+}$since it is a different distinguished trajectory from $\mathrm{DHT}_{\mathrm{W}}$, which stopped being distinguished on 11 May. Although $\mathrm{DHT}_{\mathrm{W}}$ has lost its distinguished property before we have completed our transport description, the manifold computation beyond this time still makes sense as it still is a material surface, and is still asymptotic to $\mathrm{DHT}_{\mathrm{W}}$. Although the manifold is not asymptotic to $\mathrm{DHT}_{\mathrm{W}}^{+}$, it is correct to say that $\mathrm{DHT}_{\mathrm{W}}^{+}$marks a Distinguished Trajectory on the manifold within certain accuracy $\epsilon$. Any further description of the turnstile mechanism between 11 and 26 May needs to be referred to this new DHT. This kind of replacement of the DHT of reference along the invariant manifold has been discussed in (Mancho et al., 2006). The piece of stable manifold in Fig. 8d) does not show more lobes on 26 May, and beyond this day the turnstile mechanism is disrupted due to topological transitions in the flow structure.

The altimeter-derived estimates of the velocity field have expected errors, so one question is to establish whether the information obtained from the analysis of manifolds is robust with respect to changes in the velocity field. The influence of deviations of the velocity fields on the Lagrangian structures has been examined in several works (Haller, 2002; Hernandez-Carrasco et al., 2009; Poje et al., 2009). The main conclusion of these works is that Lagrangian structures are robust with respect to errors, though conditions for the reliability of predictions are discussed. In what relates to lobes and their role in transport it is possible to say that these are formed by intersections of stable and unstable manifolds and are typically observed in the neighborhood of a Distinguished Hyperbolic Trajectory. Despite the lack of rigorous mathematical theorems for aperiodic time dependent dynamical systems it is possible to foresee a relation between the vicinity of these DHTs and chaotic saddles. The latter persist under small perturbations of the vector field, i.e. they are 

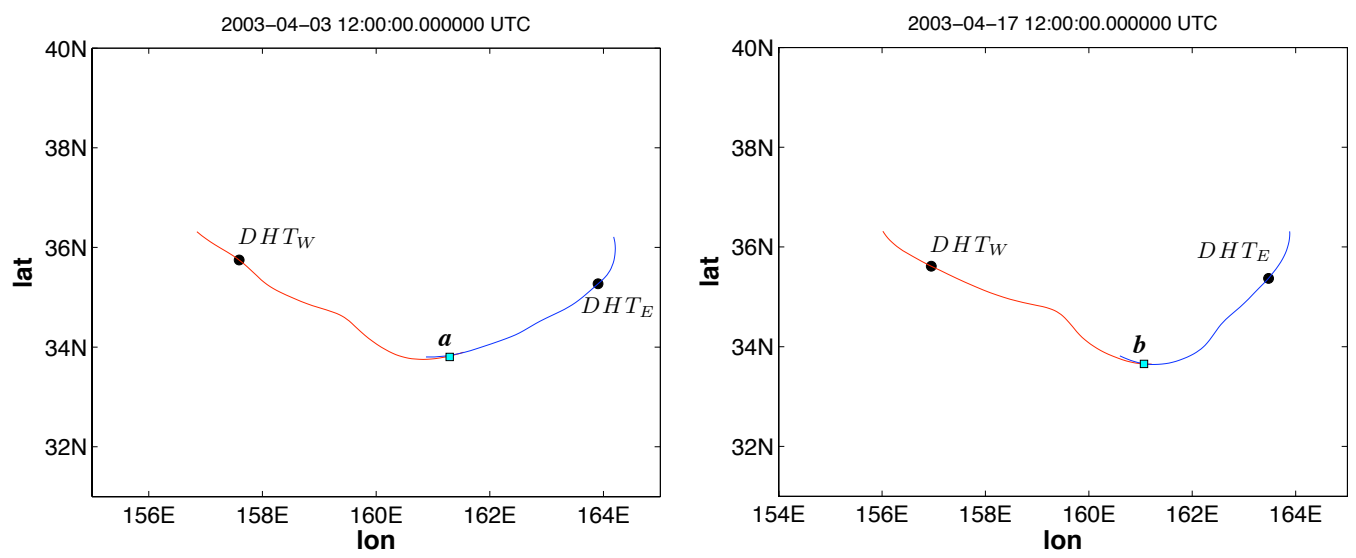

Fig. 6. Boundaries at days 3 and 17 April 2003 constructed from a (finite length) segment of the unstable manifold of DHTW and a (finite length) segment of the stable manifold of $\mathrm{DHT}_{\mathrm{E}}$. The boundary intersection points are denoted respectively by $a$ and $b$.
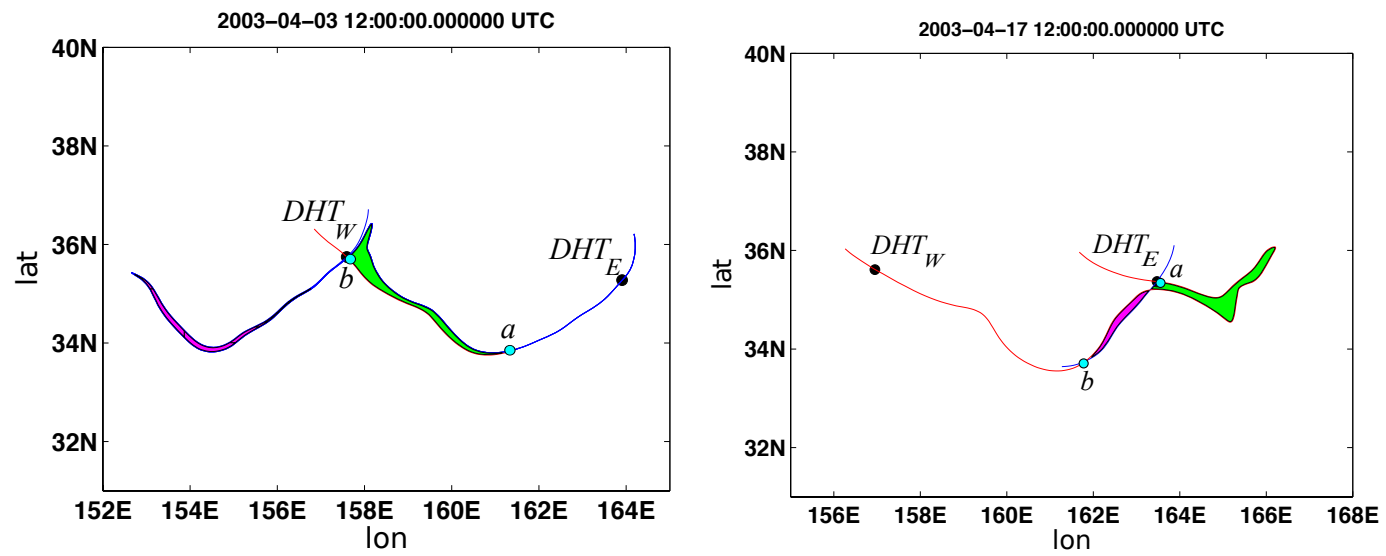

Fig. 7. Turnstile lobes at days 3 and 17 April 2003. The intersection trajectories $a$ and $b$ are displayed at both days showing their evolution from the $\mathrm{DHT}_{\mathrm{W}}$ towards the $\mathrm{DHT}_{\mathrm{E}}$. The green area evolves from north to south while the magenta area does from the south to the north.

structurally stable (Wiggins, 2003). For this reason we also expect that descriptions of the lobes near the DHTs are robust under small deviations of the measured velocity field.

\section{Conclusions}

We have explored the power of dynamical systems ideas for analyzing altimeter data sets. In particular tools for quantifying Lagrangian transport have been successfully applied to oceanographic observations. Our study analyses crossfrontal transport in altimeter data sets along a flow located in a region of the Kuroshio current. As a novelty with respect to similar studies we have identified relevant DHTs following the methodology proposed in a recent paper by Madrid and Mancho (2009), instead of using that proposed in (Ide et al., 2002; Mancho et al., 2004, 2006, 2008). We have been able to detect relevant DHTs and we confirm that DHTs hold the distinguished property only for finite time intervals. Identification and computation of DHTs is by itself an important subject, since they organize the flow in the area and, because of this and of the sensitivity of the trajectories in their neighborhood, they are candidates for launch locations in efficiently designed drifter release experiments (Poje et al., 2002; Molcard et al., 2006).

We have computed stable and unstable manifolds of the relevant DHTs. A time dependent Lagrangian "barrier" is defined from pieces of the stable and unstable manifolds and transport across it is described by lobe motion. It is found that the turnstile mechanism is at work in this observed flow, leading to a filamentous transport near the hyperbolic trajectories. This mechanism survives between 17 April 2003 and 26 May 2003. After this date transitions in the flow take place that are not well understood.

In summary this article explores the use, in a general oceanographic problem, of ideas and techniques associated with chaotic transport and dynamical systems, trying to shed some light on the mechanisms that lead to messy particle trajectories over the ocean. We expect that this tools and information will be of interest in the oceanographic community. 

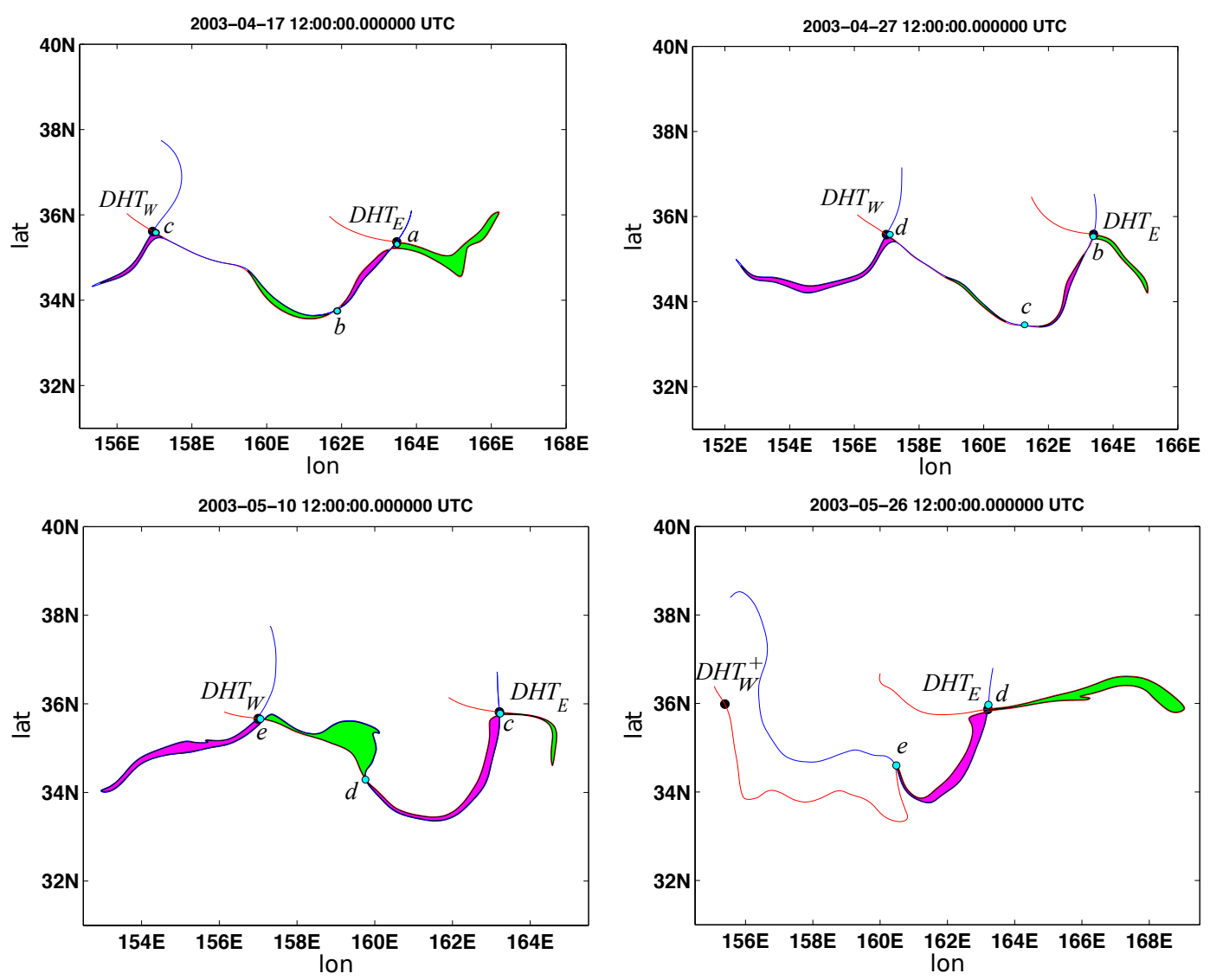

Fig. 8. Sequence of lobes between 17 April 2003 and 26 May 2003 mixing waters from north to south and viceversa at the Kuroshio current.

Acknowledgements. C. M. and A. M. M are very grateful to Antonio Turiel for his valuable help with the altimeter data. Also we are indebted with Dan Fox for his many insightful comments and suggestions.

The computational part of this work was done using the CESGA computer FINIS TERRAE.

The authors have been supported by CSIC Grant OCEANTECH No. PIF06-059, Consolider I-MATH C3-0104, MICINN Grants Nos. MTM2008-03754 and MTM2008-03840-E, and the Comunidad de Madrid Project No. SIMUMAT S-0505-ESP-0158.

Edited by: S. Wiggins

Reviewed by: J. Bruch and three other anonymous referees

\section{References}

Aurell, E., Boffetta, G., Crisanti, A., Paladin, G., and Vulpiani, A.: Predictability in the large: an extension of the concept of Lyapunov exponent, J. Phys. A, 30, 1-26, doi:10.1088/03054470/30/1/003, 1997.

Beron-Vera, F., Olascoaga, M. J., and Goni, G. J.: Oceanic mesoscale eddies as revealed by Lagrangian coherent structures, Geophys. Res. Lett., 35, L12603, doi:10.1029/2008GL033957, 2008.
Bower, A. S. and Rossby, T.: Evidence of cross-frontal exchange processes in the Gulf Stream based on isopycnal RAFOS float data, J. Phys. Oceanogr, 19(9), 1177-1190, 1989.

Bower, A. S.: A simple kinematic mechanism fro mixing fluid parcels across a meandering jet, J. Phys. Oceanogr., 21(1), 173180, 1991.

Bower, A. S., Lozier, M. S., Gary, S. F., and Böning, C. W.: Interior pathways of the North Atlantic meridional overturning circulation, Nature, 459, 243-247, doi:10.1038/nature07979, 2009.

Coulliette, C. and Wiggins, S.: Intergyre transport in a wind-driven, quasigeostrophic double gyre: An application of lobe dynamics, Nonlin. Processes Geophys., 8, 69-94, 2001, http://www.nonlin-processes-geophys.net/8/69/2001/.

Dritschel, D. G.: Contour dynamics and contour surgery: numerical algorithms for extended, high-resolution modelling of vortex dynamics in two-dimensional, inviscid, incompressible flows, Comput. Phys. Rep., 10, 77-146, 1989.

Haller, G.: Distinguished material surfaces and coherent structures in three-dimensional fluid flows, Physica D, 149, 248-277, doi:10.1016/S0167-2789(00)00199-8, 2001.

Haller, G.: Lagrangian coherent structures from approximate velocity data, Phys. Fluids, 14, 1851-1861, doi:10.1063/1.1477449, 2002.

Haller, G.: Exact theory of unsteady separation for twodimensional flows, J. Fluid Mech., 512, 257-311, doi:10.1017/S0022112004009929, 2004. 
Hernandez-Carrasco, I., Hernandez-Garcia, E., Lopez, C., and Turiel, A.: Reliability of a Lagrangian analysis from FSLEs, LAPCOD meeting 2009, Presentation B203, 2009.

Ide, K., Small, D., and Wiggins, S.: Distinguished hyperbolic trajectories in time-dependent fluid flows: analytical and computational approach for velocity fields defined as data sets, Nonlin. Processes Geophys., 9, 237-263, 2002, http://www.nonlin-processes-geophys.net/9/237/2002/.

Ju, N., Small, D., and Wiggins, S.: Existence and computation of hyperbolic trajectories of aperiodically time dependent vector fields and their approximations, Int. J. Bifurcat. Chaos, 13, 1449-1457, doi:10.1142/S0218127403007321, 2003.

Katija, K. and Dabiri, J. O.: A viscosity-enhanced mechanism for biogenic ocean mixing, Nature, 460, 624-626, doi:10.1038/nature08207, 2009.

Larnicol, G., Guinehut, S., Rio, M.-H., Drevillon, M., Faugere, Y., and Nicolas, G.: The global observed ocean products of the French Mercator project, Proceedings of the 15 years of progress in Radar altimetry, ESA symposium, Venice, March 2006.

Lavender, K. L., Davis, R. E., and Owens, W. B.: Oceanography: drifters reveal deep circulation, Nature, 407, 66-69, doi:10.1038/415954a, 2000.

Lozier, M. S., Pratt, L. J., Rogerson, A. M., and Miller, P. D.: Exchange geometry revealed by float trajectories in the Gulf Stream, J. Phys. Oceanogr., 27(11), 2327-2341, 1997.

Madrid, J. A. J. and Mancho, A. M.: Distinguished trajectories in time dependent vector fields, Chaos, 19, 013111-1-013111-18, doi:10.1063/1.3056050, 2009.

Malhotra, N. and Wiggins, S.: Geometric structures, lobe dynamics, and Lagrangian transport in flows with aperiodic timedependence, with applications to Rossby wave flow, J. Nonlinear Sci., 8, 401-456, doi:10.1007/s003329900057, 1998.

Mancho, A. M., Small, D., Wiggins, S., and Ide, K.: Computation of stable and unstable manifolds of hyperbolic trajectories in two-dimensional, aperiodically time-dependent vector fields, Physica D, 182, 188-222, doi:10.1016/S0167-2789(03)00152-0, 2003.

Mancho, A. M., Small, D., and Wiggins, S.: Computation of hyperbolic trajectories and their stable and unstable manifolds for oceanographic flows represented as data sets, Nonlin. Processes Geophys., 11, 17-33, 2004, http://www.nonlin-processes-geophys.net/11/17/2004/.

Mancho, A. M., Small, D., and Wiggins, S.: A tutorial on dynamical systems concepts applied to Lagrangian transport in oceanic flows defined as finite time data sets: theoretical and computational issues, Phys. Rep., 437(3-4), 55-124, doi:10.1016/j.physrep.2006.09.005, 2006.

Mancho, A. M., Small, D., and Wiggins, S.: A comparison of methods for interpolating chaotic flows from discrete velocity data, Comput. Fluids, 35, 416-428, doi:10.1016/j.compfluid.2005.02.003, 2006.
Mancho, A. M., Hernández-García, E., Small, D., and Wiggins, S.: Lagrangian transport through an ocean front in the Northwestern Mediterranean sea, J. Phys. Oceanogr., 38, 1222, doi:10.1175/2007JPO3677.1, 2008.

Mendoza, C. and Mancho, A. M.: The hidden geometry of ocean flows, Chaotic Dynamics, arXiv:1003.0368, submitted, 2010.

Molcard, A., Poje, A., and Özgökmen, T.: Directed drifter launch strategies for Lagrangian data assimilation using hyperbolic trajectories, Ocean Model., 12, 268-289, doi:10.1016/j.ocemod.2005.06.004, 2006.

Nese, J. M.: Quantifying local predictability in phase space, Physica D, 35, 237-250, doi:10.1016/0167-2789(89)90105-X, 1989.

Poje, A. C., Toner, M., Kirwan, A. D., and Jones, C. K. R. T.: Drifter launch strategies based on Lagrangian templates, J. Phys. Oceanogr., 32(6), 1855-1869, doi:10.1175/1520-0485, 2002.

Poje, A., Haza, A., and Ozgokmen, T.: Resolution and Scale Dependence of Relative Dispersion in a Hierarchy of Ocean Models, LAPCOD meeting 2009, Presentation B101, 2009.

Press, W. H., Teukolsky, S. A., Vetterling, W. T., and Flannery, B. P.: Numerical recipes in C, Cambridge University Press, United Kingdom, 1992.

Rio, M.-H. and Hernandez, F: A mean dynamic topography computed over the world ocean from altimetry, in situ measurements, and a geoid model, J. Geophys. Res.-Oceans, 109, C12032, doi:10.1029/2003JC002226, 2004.

Rio, M.-H. and Hernandez, F.: High-frequency response of wind-driven currents measured by drifting buoys and altimetry over the world ocean, J. Geophys. Res., 108(C8), 3283, doi:10.1029/2002JC001655, 2003.

Rio, M.-H., Schaeffer, P., Hernandez, F., and Lemoine, J.: The estimation of the ocean Mean Dynamic Topography through the combination of altimetric data, in-situ measurements and GRACE geoid: From global to regional studies. Proceedings of the GOCINA International Workshop, Luxembourg, 2005.

Rom-Kedar, V., Leonard, A., and Wiggins, S.: An analytical study of transport, mixing, and chaos in an unsteady vortical flow, J. Fluid Mech., 214, 347-394, doi:10.1017/S0022112090000167, 1990.

Rossi, V., López, C., Sudre, J., Hernández-García, E., and Garçon, V.: Comparative study of mixing and biological activity of the Benguela and Canary upwelling systems, Geophys. Res. Lett., 35, L11602, 1-5, 2008.

Rypina, I. I., Brown M. G., and Kocak, H.: Transport in an idealized three-gyre system with application to the Adriatic Sea, J. Phys. Oceanogr. 39, 675-690, 2009.

Waseda, T., Mitsudera, H., Taguchi, B., and Yoshikawa, Y.: On the eddy-Kuroshio interaction: meander formation process, J. Geophys. Res., 108(C7), 3220, doi:10.1029/2002JC001583, 2003.

Wiggins, S.: Introduction to Applied Nonlinear Dynamical Systems and Chaos, Springer Verlag, 2003. 